LUPUS NEPHRITIS

\section{Oncogenes and inflammasomes in lupus nephritis}

Activation of the NLRP3

inflammasome contributes to the pathogenesis of lupus nephritis, a serious manifestation of systemic lupus erythematosus (SLE). A new study reveals how the oncogene serine/threonine-protein kinase PIM1 interacts with the NLRP3 inflammasome to contribute to kidney damage in SLE.

PIM1 is known to modulate regulatory $\mathrm{T}$ cell differentiation and $B$ cell survival, both of which are involved in the pathogenesis of SLE. PIM1 is also known to enhance the activity of the transcription factor NFATc1, which is the target of calcineurin inhibitors, drugs used to treat lupus nephritis. However, little is known about how PIM1 interacts with the NLRP3 inflammasome.

To investigate the role of PIM1 in lupus nephritis, the researchers used two different types of lupus-prone mice. Inhibition of PIM1 using
AZD1208 reduced proteinuria and the severity of glomerulonephritis in NZB/W F1 mice compared with untreated mice, results that were recapitulated in MRL/lpr mice treated with the PIM1 inhibitor SMI-4a. PIM1 inhibition also reduced the expression of NFATc1, NLRP3 and caspase- 1 in the kidneys of NZB/W F1 mice compared with untreated mice.

In vitro studies using human podocytes revealed that small interfering RNA inhibition of PIM1 reduced autoantibodyinduced expression of NFATc1 and activation of the NLRP3 inflammasome. Interestingly, PIM1 inhibition stopped the increase in intracellular calcium concentrations caused by autoantibody stimulation. Calcium is a known activator of inflammasome signalling, suggesting that PIM1 might regulate
Inhibition of PIM1 using AZD1 208 reduced proteinuria and the severity of glomerulonephritis

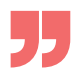

\section{OSTEOARTHRITIS}

\section{mTORC1 promotes pathological bone-cartilage interplay}

Aberrant subchondral bone remodelling is implicated in the development of osteoarthritis (OA), but how this process is induced and how increased bone turnover promotes articular cartilage degeneration is unclear. New findings implicate pre-osteoblast expansion and secretion of the chemokine CXCL12 mediated by the cellular energy sensor mTORC1.

"Previous studies by our group and others have shown that balanced mTORC 1 activity is critical for bone metabolism and development," explains corresponding author Xiaochun Bai. "However, the role of mTORC1 in subchondral bone formation during the pathogenesis of OA and the underlying mechanisms have not been reported."

The researchers used an inducible conditional-knockout approach to generate mice (referred to hereafter as $\triangle \mathrm{TSC} 1$ mice) that lack TSC1 (a negative regulator of $\mathrm{mTORC} 1$ ) in pre-osteoblasts after doxycycline treatment, resulting in constitutive activation of $\mathrm{mTORC} 1$ in these cells.

$\triangle \mathrm{TSC} 1$ mice had abnormal bone formation and bone sclerosis 6 weeks after doxycycline-treatment but their articular cartilage integrity was intact. However, in an anterior cruciate ligament transection (ACLT) model of post-traumatic OA, TSC1 deletion accelerated cartilage damage and OA development.

By contrast, disrupting mTORC1 activation in pre-osteoblasts of mice (through deletion of the mTORC1 component RAPTOR) reduced subchondral bone formation and articular cartilage degeneration in the ACLT model.

Interestingly, the pre-osteoclasts from $\triangle \mathrm{TSC} 1$ mice had increased expression of CXCL12, and treatment with an

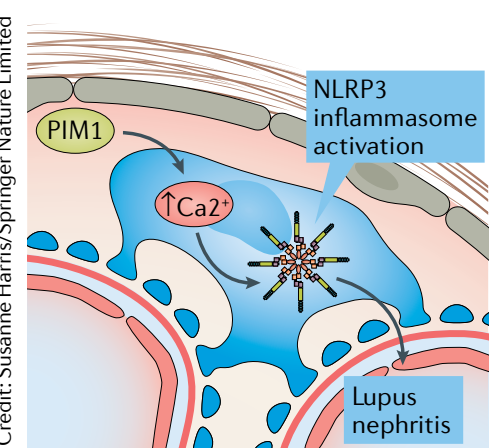

NLRP3 inflammasome activation by controlling calcium flux.

"PIM1 participates in the pathogenesis of lupus nephritis by regulating both the NLRP3 inflammasome and NFATc1," comments corresponding author Jijun Zhao. "Theoretically, PIM1 inhibitors might be superior to calcineurin inhibitors in lupus nephritis as they can inhibit both NLRP3 and NFATc1. However, further studies are needed to answer this question."

Joanna Collison

ORIGINAL ARTICLE Fu, R. et al. Pim-1 as a therapeutic target in human lupus nephritis. Arthritis Rheumatol. https://doi.org/10.1002/ art.40863 (2019)

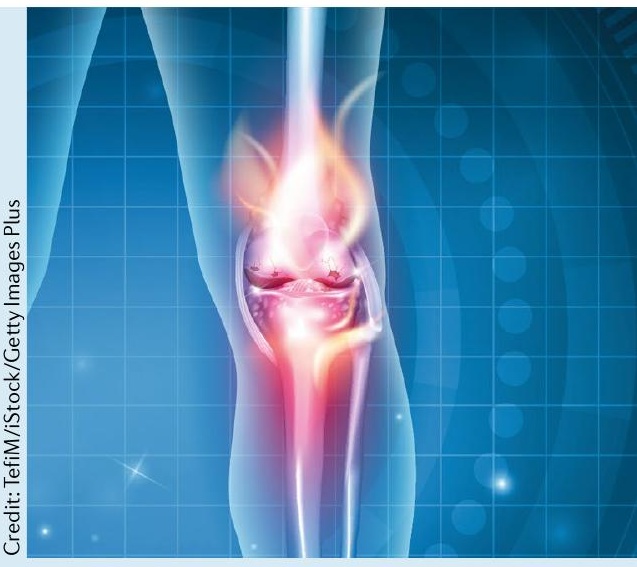

anti-CXCL12 antibody attenuated ACLT-induced articular cartilage degeneration in $\triangle \mathrm{TSC} 1$ mice.

"We have shown that pre-osteoblasts of subchondral bone might be another major source of abnormally increased CXCL12 expression in OA in addition to synovial tissue," says Bai. "CXCL12 might target chondrocytes through the circulation to promote OA."

Jessica McHugh

ORIGINAL ARTICLE Lin, C. et al. Activation of mTORC 1 in subchondral bone preosteoblasts promotes osteoarthritis by stimulating bone sclerosis and secretion of CXCL12. Bone Res. 7, 5 (2019) 